\title{
ARTICLE OPEN \\ Searching for topological Fermi arcs via quasiparticle interference on a type-II Weyl semimetal $\mathrm{MoTe}_{2}$
}

\author{
Davide laia ${ }^{1}$, Guoqing Chang $\mathbb{D D}^{2}$, Tay-Rong Chang ${ }^{3}$, Jin Hu $\mathbb{D}^{4}$, Zhiqiang Mao ${ }^{4}$, Hsin Lin ${ }^{2}$, Shichao Yan ${ }^{5}$ and Vidya Madhavan ${ }^{1}$
}

Weyl semimetals display a novel topological phase of matter where the Weyl nodes emerge in pairs of opposite chirality and can be seen as either a source or a sink of Berry curvature. The exotic effects in Weyl semimetals, such as surface Fermi arcs and the chiral anomaly, make them a new playground for exploring novel functionalities. Further exploiting their potential applications requires clear understanding of their topological electronic properties. Here we report a Fourier transform scanning tunneling spectroscopy (FT-STS) study on a type-II Weyl semimetal candidate MoTe $\mathrm{H}_{2}$ whose Weyl points are predicated to be located above Fermi level. Although its electronic structure below the Fermi level has been identified by angle resolved photo emission spectroscopy, by comparing our experimental data with first-principles calculations, we are able to identify the origins of multiple scattering channels both below and above Fermi level. Our calculations also show the existence of both trivial and topological arc-like states above the Fermi energy. In the FT-STS experiments, we have observed strong signals from intra-arc scatterings as well as from the scattering between the arc-like surface states and the projected bulk states. A detailed comparison between our experimental observations and calculated results reveals the trivial and non-trivial scattering channels are difficult to distinguish in this compound. Interestingly, we find that the broken inversion symmetry changes the terminating states on the two inequivalent surfaces, which in turn changes the relative strength of the scattering channels observed in the FT-STS images on the two surfaces.

npj Quantum Materials (2018)3:38; doi:10.1038/s41535-018-0112-5

\section{INTRODUCTION}

Transition metal dichalcogenides (TMDs) provide a fertile ground for exploring exotic collective quantum phenomena, such as superconductivity and charge density waves. ${ }^{1-3}$ Recently, the $\mathrm{Mo}_{x} \mathrm{~W}_{1-x} \mathrm{Te}_{2}$ class of TMDs has attracted great interest due to the topological nature of the electronic states and as a topological Weyl semimetal (TWS). ${ }^{4-7}$ Their low-energy excitations behave as Weyl fermions that always appear in pairs with opposite chirality. TWSs are classified into type-I and type-II: type-I TWSs have point like Fermi surfaces and respect Lorentz invariance, while type-II with tilted Weyl cones break Lorentz invariance. ${ }^{5,8,9}$ Type-I TWSs were first predicted and experimentally discovered in the $(\mathrm{Ta}, \mathrm{Nb})$ (As, P) family compounds. ${ }^{8-11}$ Type-II TWSs have been proposed to exist in the $\mathrm{Mo}_{x} \mathrm{~W}_{1-x} \mathrm{Te}_{2}$ family $\mathrm{TMDs}^{4-7}$ and LaAlGe. ${ }^{12}$ In this system, the tilted Weyl cones that arise from topologically protected crossings of valence and conduction bands cause touching points between electron and hole pockets near the Fermi level. Exotic Fermi arcs states that join these touching points, are expected on the surfaces of these materials. In the last few years, there have been a series of experimental studies aimed at verifying the existence of the Fermi arcs. $^{13-21}$

For the $\mathrm{Mo}_{x} \mathrm{~W}_{1-x} \mathrm{Te}_{2}$ family, the Weyl nodes are usually located at energies above the Fermi level which makes it difficult to be accessed by conventional angle resolved photo emission spectroscopy (ARPES). ${ }^{13-15,17,20}$ Fourier transform scanning tunneling spectroscopy (FT-STS) contains information on the scattering vectors between electronic states in momentum space and can provide the information on both the occupied and unoccupied states $^{22}$ which makes it a particularly powerful probe for the topological states of the $\mathrm{Mo}_{x} \mathrm{~W}_{1-x} \mathrm{Te}_{2}$. There have been several FTSTS studies on the $\mathrm{Mo}_{x} \mathrm{~W}_{1-x} \mathrm{Te}_{2}$. $^{15,23-28}$ However, due to the complex band structure and the low signal strength in the FT-STS images, the existence of Fermi arcs in $\mathrm{Mo}_{x} \mathrm{~W}_{1-x} \mathrm{Te}_{2}$ system still remains to be clarified.

Here we use low-temperature scanning tunneling microscopy (STM) and first-principles calculations to directly visualize and identify the electronic states in $\mathrm{MoTe}_{2}$, an end member of the $\mathrm{Mo}_{x} \mathrm{~W}_{1-x} \mathrm{Te}_{2}$ system. The other end member, $\mathrm{WTe}_{2}$ was the first predicated type-II Weyl semimetal, but the small momentum separation between the opposite Weyl points that are located about $50 \mathrm{mV}$ above the Fermi level, made experimental confirmation by conventional ARPES difficult. ${ }^{5,14} \mathrm{MoTe}_{2}$ was later predicated as another candidate of type-II Weyl semimetal where the Weyl points have six times larger momentum separation which makes experimental probe of Weyl nodes much easier. ${ }^{6,7}$ For $\mathrm{MoTe}_{2}$, previous ARPES works have found evidence for the

\footnotetext{
${ }^{1}$ Department of Physics and Frederick Seitz Materials Research Laboratory, University of Illinois Urbana-Champaign, Urbana, IL 61801, USA; ${ }^{2}$ Institute of Physics, Academia Sinica, Taipei 11529, Taiwan; ${ }^{3}$ Department of Physics, National Cheng Kung University, Tainan 701, Tainan, Taiwan; ${ }^{4}$ Department of Physics and Engineering Physics, Tulane University, New Orleans, LA 70018, USA and ${ }^{5}$ School of Physical Science and Technology, ShanghaiTech University, Shanghai 201210, China Correspondence: Shichao Yan (yanshch@shanghaitech.edu.cn) or Vidya Madhavan (vm1@illinois.edu)
} 

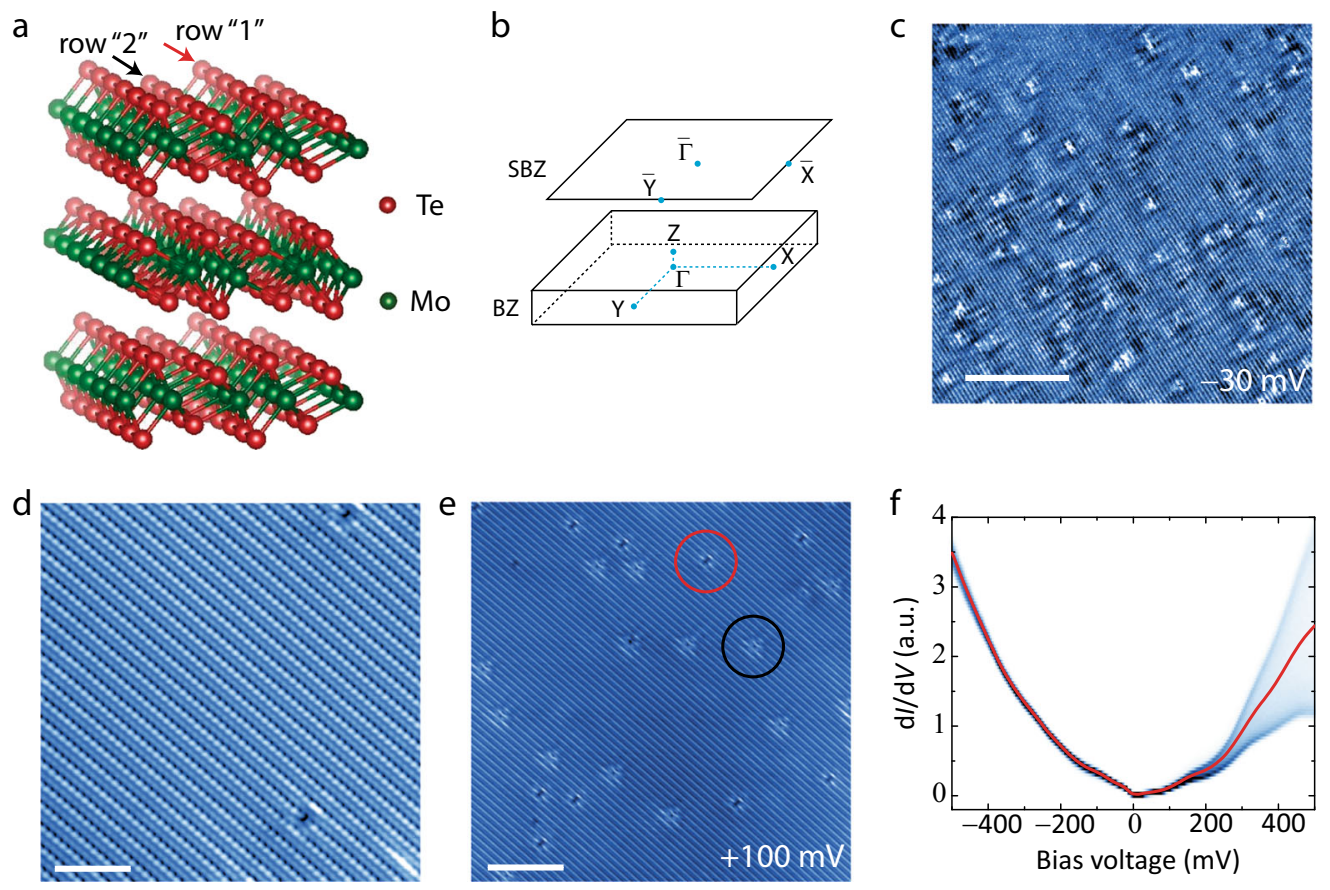

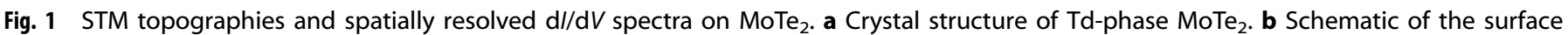
Brillouin zones (SBZ) and bulk Brillouin zones (BZ) of MoTe . $\mathbf{c} \mathrm{d} / / \mathrm{dV}$ map at $-30 \mathrm{mV}$. The scale bar shown represents $15 \mathrm{~nm}$. $\mathbf{d}$ High-resolution STM topography taken with $+100 \mathrm{mV}$ and $150 \mathrm{pA}$. The scale bar shown represents $3 \mathrm{~nm}$. e Large-scale STM topographies taken with $+100 \mathrm{mV}$ and $300 \mathrm{pA}$. The black and red circles indicate two different kinds of atomic impurities on the top Te-layer. The scale bar shown represents $10 \mathrm{~nm}$. f Spatially resolved $\mathrm{d} / / \mathrm{d} V$ spectra (blue) taken over a $50 \mathrm{~nm}$ by $50 \mathrm{~nm}$ area with $-100 \mathrm{mV}$ and 750 pA setpoint. The red curve is the averaged $\mathrm{d} / / \mathrm{d} V$ spectrum over this area
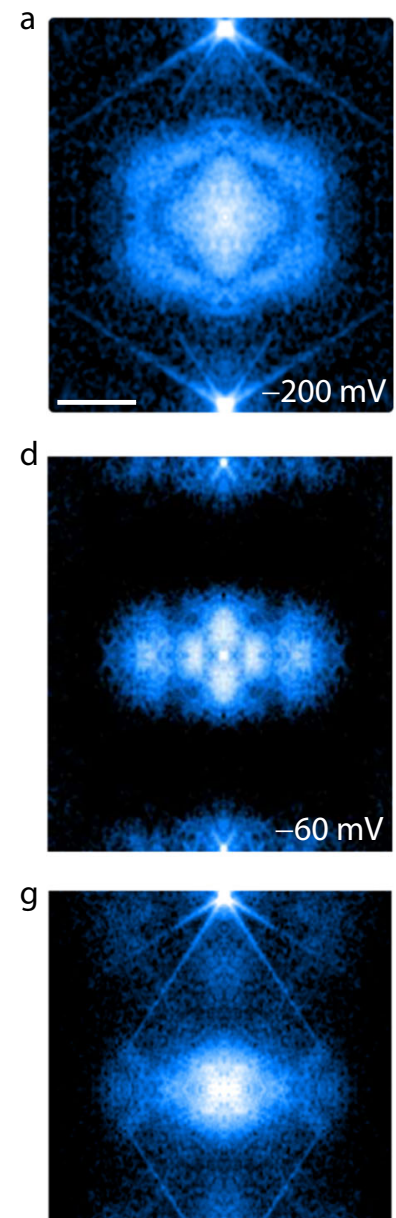
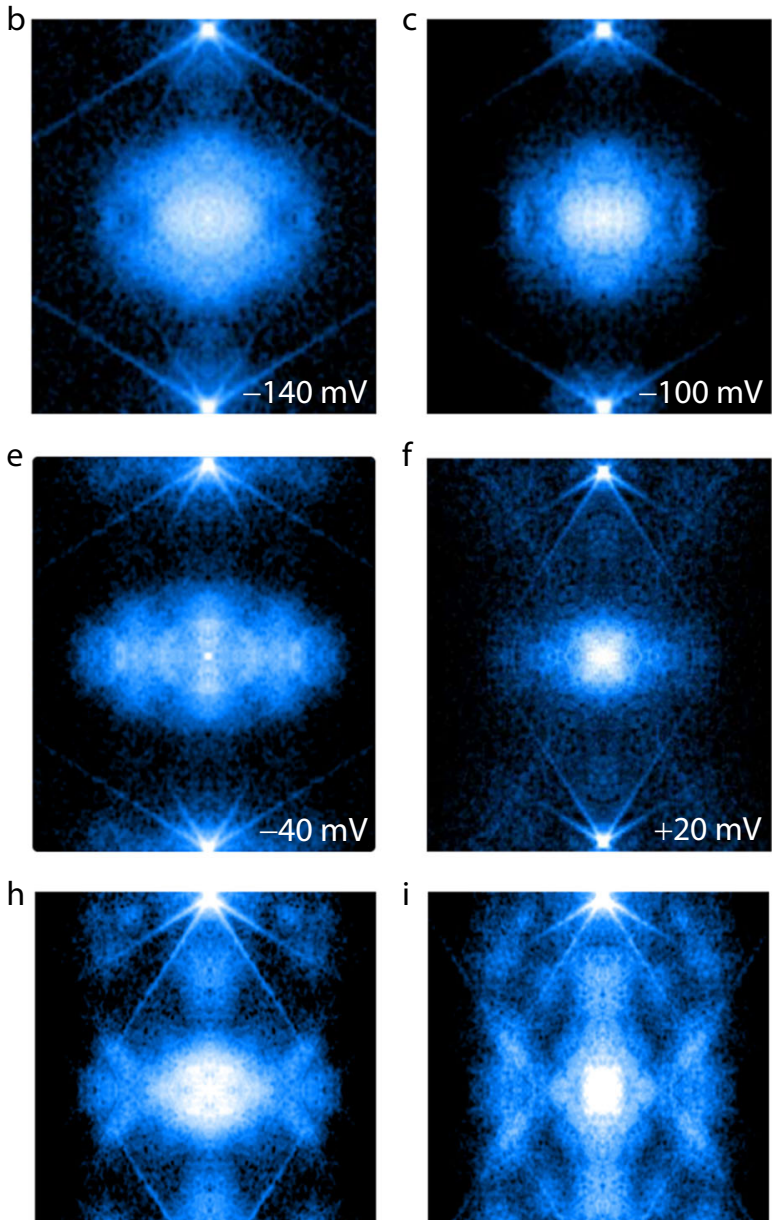
Fig. 2 Quasiparticle interference on MoTe 2 . a-i Mirror-symmetrized FT-STSs at energies of $-200 \mathrm{mV},-140 \mathrm{mV},-100 \mathrm{mV},-60 \mathrm{mV},-40 \mathrm{mV}$, $+20 \mathrm{mV},+40 \mathrm{mV},+60 \mathrm{mV}$, and $+100 \mathrm{mV}$, respectively. For the FT-STSs with positive sample bias voltages, before performing the Fourier transform, the $d / / d V$ maps are first normalized with the tunneling current map at $+200 \mathrm{mV}$. The scale bar shown represents $0.4 \AA^{-1}$

4

Constant energy contour

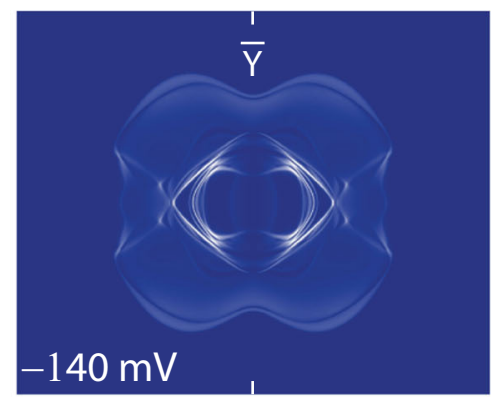

$\mathrm{b}$

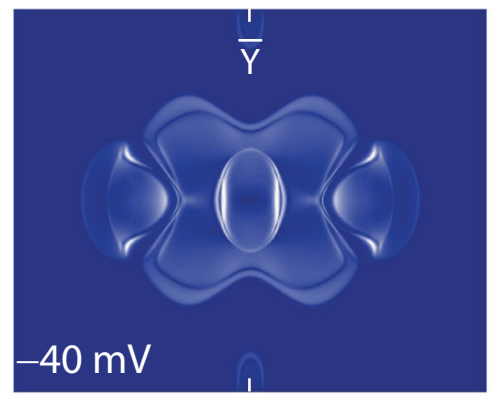

C

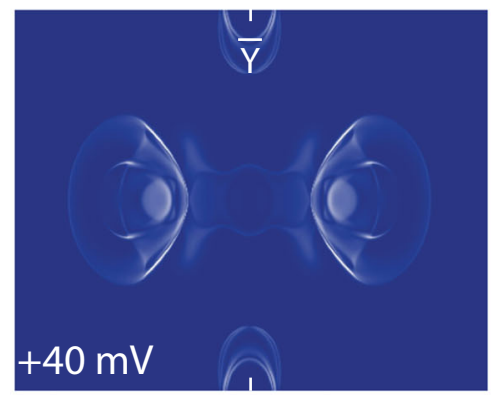

d

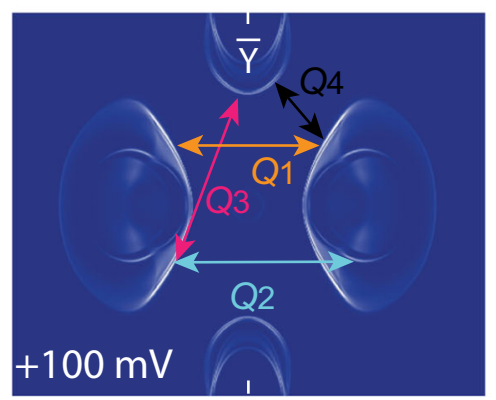

Calculated FT-STS
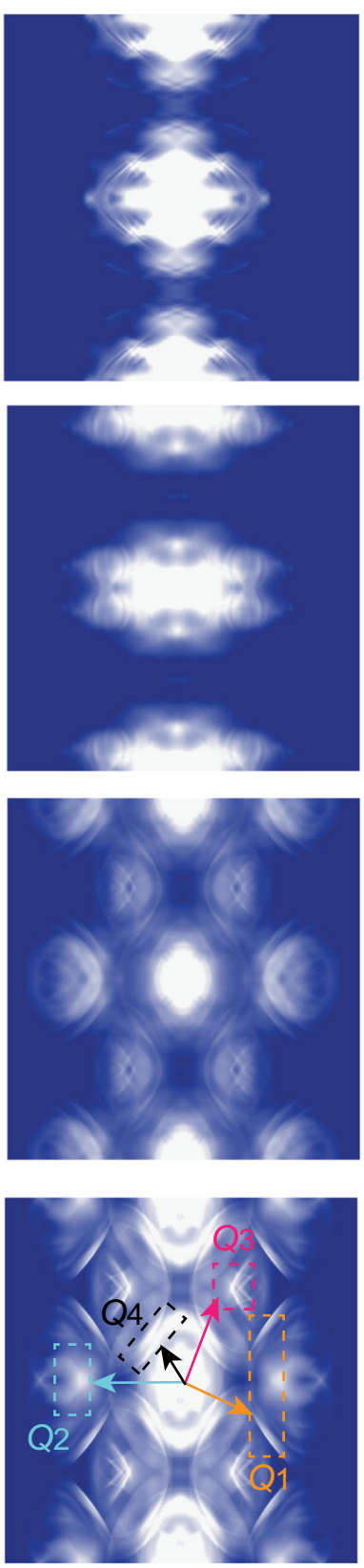

Measured FT-STS
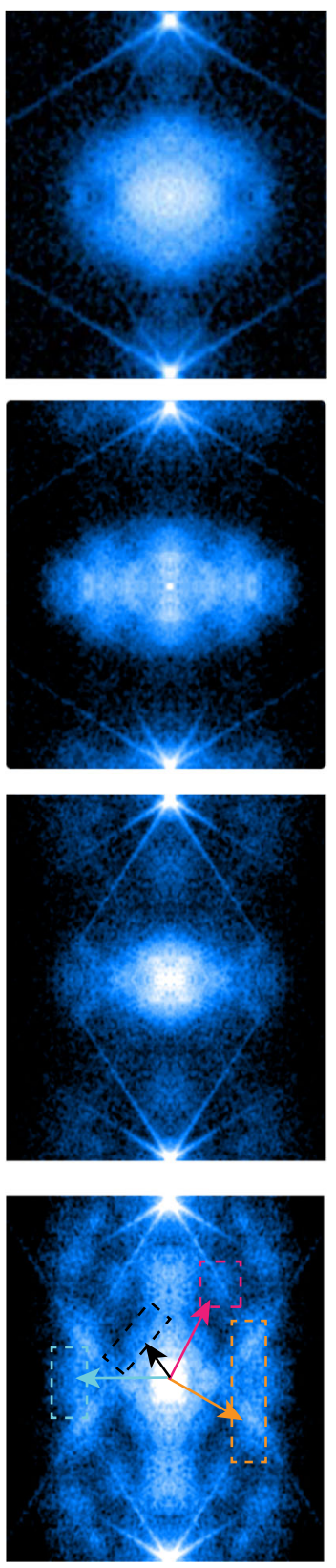

Fig. 3 Comparison between the calculated FT-STSs and the measured FT-STSs. a Constant energy contours of MoTe $e_{2}$ at the energy $E=$ $-140 \mathrm{mV}$ (left panel); theoretically simulated FT-STS at $-140 \mathrm{mV}$ (middle panel); experimentally measured FT-STS at $-140 \mathrm{mV}$ (right panel). b, c The same as a, but for energy $E=-40 \mathrm{mV}$ and $+40 \mathrm{mV}$, respectively. $\mathbf{d}$ The same as a-c, but for energy $E=+100 \mathrm{mV} . Q_{1}, Q_{2}, Q_{3}$ and $Q_{4}$ are the scattering vectors (see the main text for details)

trivial and topologically protected Fermi arcs at energies below Fermi level. ${ }^{18-20}$ However, for various reasons such as the size of the STS maps or the properties of the scattering impurities, previous FT-STSs on $\mathrm{MoTe}_{2}$ did not show sufficient signal strength to perform a clear analysis of the FT-STS data, and have not arrived at an unequivocal conclusion about the nature of the Weyl states. In this work, we present FT-STS data with much higher signal strength that allows us to compare the various scattering channels observed in the experimental data with theory, and reach a robust conclusion on the origins of the scattering channels. Interestingly, based on this higher resolution FT-STS data, we come to different conclusions compared to the previous work: ${ }^{15,23,24,26}$ the trivial and non-trivial scattering channels are indistinguishable in the FTSTS images. Moreover, the broken inversion symmetry in this 
compound is reflected in the FT-STS images taken on two inequivalent surfaces.

\section{RESULTS}

The $T_{\mathrm{d}}$ phase crystal has an orthorhombic structure with van der Waals stacking of Te-Mo-Te sandwich layers along the $c$-axis direction as shown in Fig. 1a. Due to the weak van der Waals interaction between the Te layers, the $\mathrm{MoTe}_{2}$ sample cleaves at the Te layers and the cleaved surface is Te-terminated. The bulk, and surface Brillouin zones for this termination, is shown in Fig. 1b. In high-resolution STM topographies, the two inequivalent Te atomic rows in the top Te layer can be clearly resolved (Fig. 1d). There are mainly two kinds of atomic defects and they look different in the STM topographies taken with positive bias voltages (as shown in the red and black circles in Fig. 1e). The defects lie on the Te sites and are likely to be Te vacancies/ impurities in the two Te atomic rows at the top Te layer. In addition, in the negative bias topography, there are other faint defects that may be attributed to vacancies/impurities in the layers underneath (more details can be found in Supplementary Fig. S1 and Fig. S2).

Figure $1 \mathrm{f}$ shows the position dependent $\mathrm{d} / \mathrm{d} V$ spectra taken on a $50 \mathrm{~nm}$ by $50 \mathrm{~nm}$ area on $\mathrm{MoTe}_{2}$ in the energy range of $\pm 500 \mathrm{mV}$. In general, the $\mathrm{d} l / \mathrm{d} V$ spectra are parabola-shaped with minimum around $10 \mathrm{mV}$ above the Fermi level. Despite the presence of impurities, the low energy density of states as well as the overall parabola-shape of the spectra remains homogeneous and there are no clear impurity-induced resonance states in this energy range. ${ }^{26}$ However, the scattering pattern of quasiparticles by the atomic impurities can be clearly seen in the $d / / d V$ maps, Fig. 1c. The next step is to study the Fourier transforms of the $\mathrm{d} / / \mathrm{d} V$ maps to look for signatures of the electronic states in momentum space.

Figure 2a-i shows FT-STS images at a few energies below and above the Fermi level (also see Figs. S6 and S7). There is a distinct change in the FT-STS as we go from negative to positive energies. With increasing the bias above the Fermi level, several strong scattering vectors gradually emerge. To identify the origin of the scattering vectors we carried out first-principles density functional theory (DFT) calculations of band structures of $\mathrm{MoTe}_{2}$ which were then used to calculate FT-STS images. Using the iterative Green's function method, we obtained the surface states of $\mathrm{MoTe}_{2}$ under different terminations (details in Supplementary Section 8 which were then used to simulate the FT-STS patterns using spindependent scattering probability method).

As can be seen in Fig. 3, the simulated FT-STS matches well with the measured FT-STS over a wide range of energies. In the measured FT-STS at energies below $-100 \mathrm{mV}$ (right panel in Fig. $3 a)$, there is a lip-like feature with a bright center. By comparing with the simulated FT-STS at $-140 \mathrm{mV}$, we conclude that this liplike feature is due to the scattering of the bulk states of $\mathrm{MoTe}_{2}$ (Supplementary Fig. S5). At $-40 \mathrm{mV}$, the measured FT-STS shows two short arc-like features at the left or right side of the bright center spot (right panel of Fig. 3b). Due to the multiple scattering vectors seen in the simulated FT-STS at negative bias voltages, it is difficult to unambiguously identify the origin of these two short arcs. However, by comparing the momentum-space position of these short arcs, we conclude that they most likely arise from the scattering processes from the trivial surface states to the holepockets. At positive bias voltages, the scattering patterns become much clearer (Fig. 2i). By comparing the calculated FT-STS with the measured FT-STS at $+40 \mathrm{mV}$ and $+100 \mathrm{mV}$ (Fig. 3c, $\mathrm{d}$ and Supplementary Fig. S9), we can for the first time identify all the scattering patterns as: $Q_{1}$ is induced by the intra-scattering of the topologically trivial surface states; $Q_{2}$ is caused by the scattering between the surface states and the electron pockets. $Q_{3}$ and $Q_{4}$ are due to the scattering between the surface states and the projected bulk states at the $\bar{Y}$ point.
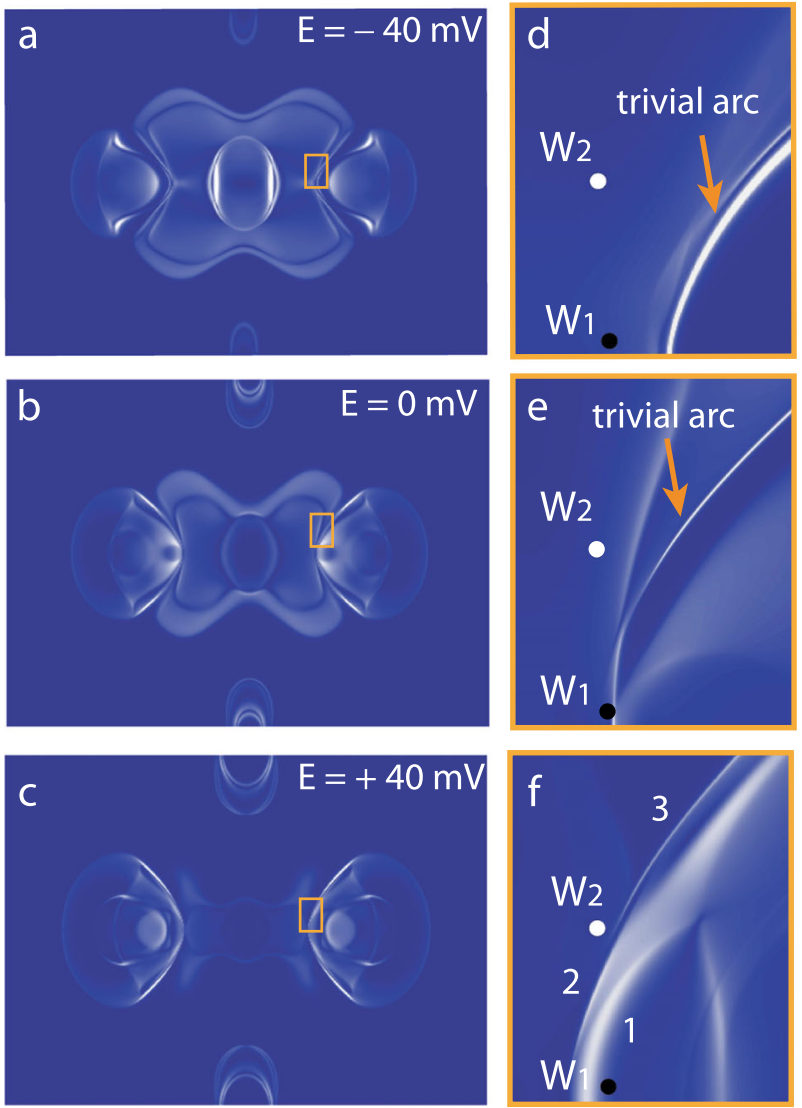

Fig. 4 Trivial Fermi arcs and Weyl nodes. a-c Calculated constant energy contours of $\mathrm{MoTe}_{2}$ at the energy $E=-40 \mathrm{mV}, 0 \mathrm{mV}$, and $+40 \mathrm{mV}$, respectively. $\mathbf{d}-\mathbf{f}$ The zoom-in of the orange box in $\mathbf{a}, \mathbf{b}$, and c. The long arc away from the Weyl nodes (black and white dots labeled as $W_{1}$ and $W_{2}$ ) is topologically trivial. The trivial arc indicated by the orange arrows in $\mathbf{d}$ and $\mathbf{e}$ is cut into three pieces, labeled as " 1 ", " 2 " and " 3 " in $\mathbf{f}$, where " 2 " is the topological Fermi arc. The size of d-f is about $0.08 \AA^{-1}$ by $0.1 \AA^{-1}$

Although, the high-resolution FT-STS data clearly show multiple scattering vectors from the surface states and our experimental measurements match the numerical calculations, these data alone do not provide unambiguous evidence for the existence of nontrivial Fermi arcs. According to our calculations, the Weyl points for $\mathrm{MoTe}_{2}$ are located at $4 \mathrm{mV}$ and $57 \mathrm{mV}$. As the energy increased from the Fermi level to $+100 \mathrm{mV}$, the scattering vectors $\left(Q_{1}-Q_{4}\right)$ gradually emerge in the measured FT-STSs (Fig. $2 \mathrm{f}-\mathrm{i}$ ), and there is no sudden change in the FT-STSs near the energy positions of the Weyl nodes. This suggests that there are no clear features induced by the non-trivial Fermi arcs in the FT-STSs on $\mathrm{MoTe}_{2}$. We note that our conclusions are in contrast with previous studies. ${ }^{15,23,24,26,27}$ Most previous STM studies have claimed that $\mathrm{Mo}_{x} \mathrm{~W}_{1-x} \mathrm{Te}_{2}$ family material is a Weyl semimetal based on the existence of the long Fermi arcs in the FT-STS data. However, based on our theoretical and experimental studies we find that the long arcs arise primarily from topologically trivial scattering channels. In addition, for $\mathrm{Mo}_{0.66} \mathrm{~W}_{0.34} \mathrm{Te}_{2}$ samples, the absence of the scattering vector $\left(Q_{3}\right)$ between the arc-like feature and projected bulk states was taken as proof of the existence of the topological Fermi arc. ${ }^{27}$ In contrast, in our high-resolution data on $\mathrm{MoTe}_{2}$, we clearly detect this scattering vector $\left(Q_{3}\right)$ thereby determining that the scattering process from trivial surface states forms an important component of the scattering seen in our FTSTS data. 

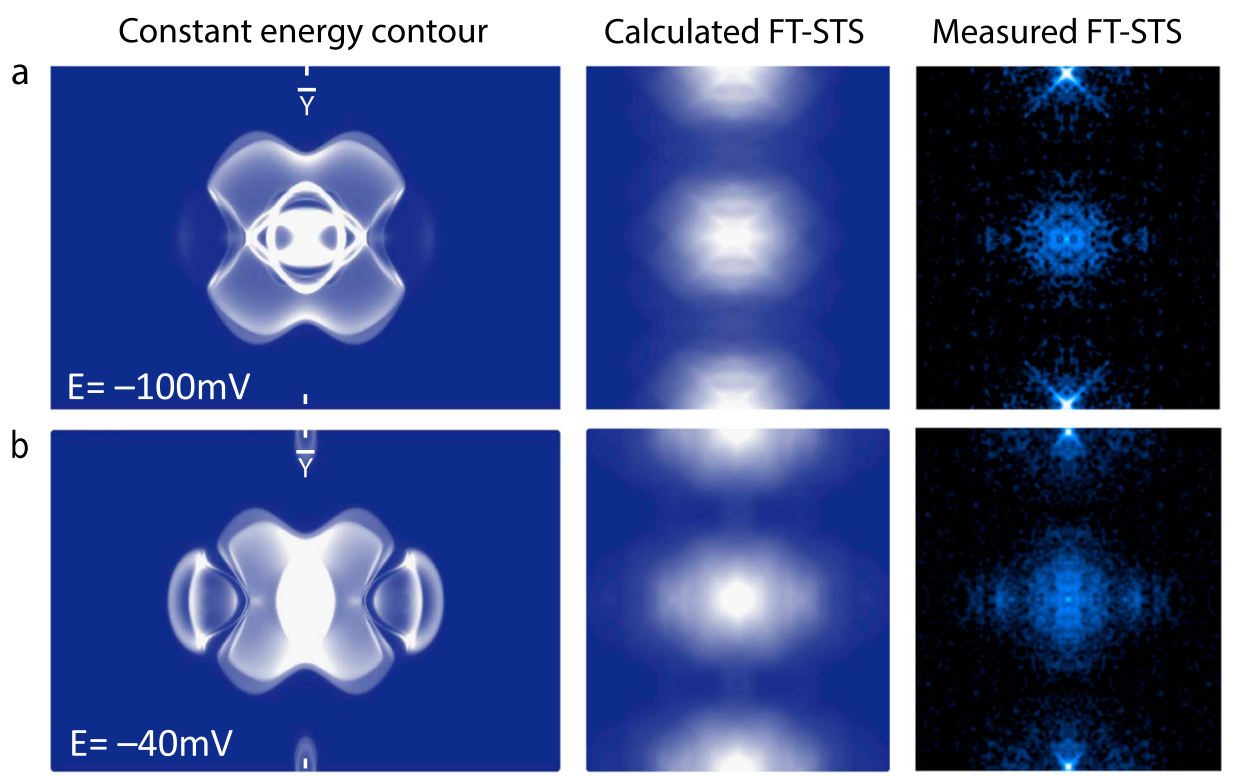

C
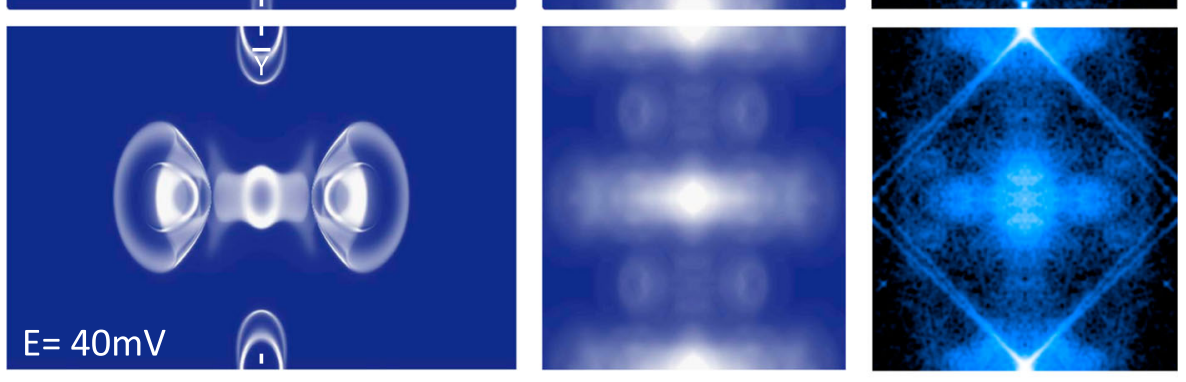

$\mathrm{d}$
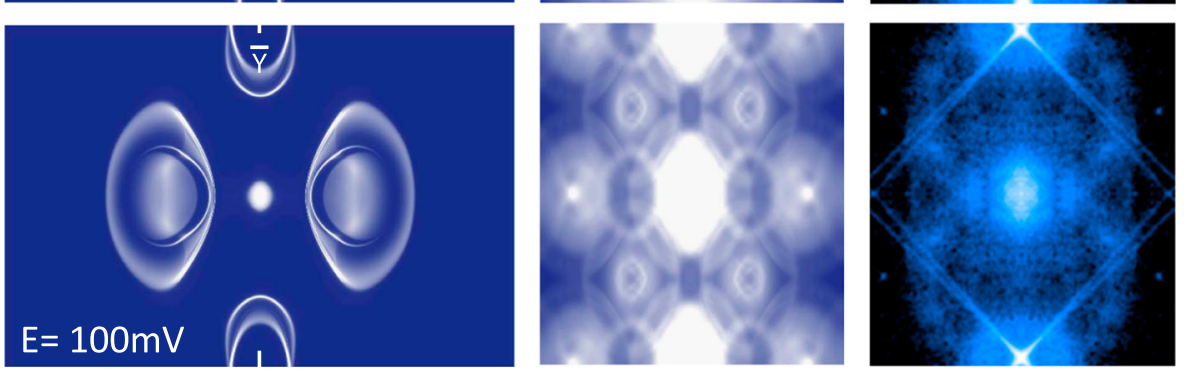

Fig. 5 Calculated and measured FT-STSs on the second, inequivalent broken inversion symmetry surface. a Constant energy contours of $\mathrm{MoTe}_{2}$ at the energy $E=-100 \mathrm{mV}$ (left panel); theoretically simulated FT-STS at $-100 \mathrm{mV}$ (middle panel); experimentally measured FT-STS at $-100 \mathrm{mV}$ (right panel). b-d The same as a, but for energy $E=-40 \mathrm{mV},+40 \mathrm{mV}$, and $+100 \mathrm{mV}$, respectively

\section{DISCUSSION}

This leads to the question: why is it difficult to detect the nontrivial Fermi arcs in $\mathrm{MoTe}_{2}$ even with high-resolution FT-STS measurements? Weyl fermions are local singularities in momentum space (Fig. S10). Therefore, at energies or momentum space away from the Weyl nodes, the non-trivial properties induced by Weyl fermions are negligible. In Fig. 4 we show the calculated surface states of $\mathrm{MoTe}_{2}$ at three different energies, below, at, and above the Fermi level. Figure $4 a-c$ shows constant energy contours at $-40 \mathrm{mV}, 0 \mathrm{mV}$, and $+40 \mathrm{mV}$, respectively. Figure $4 \mathrm{~d}-\mathrm{f}$ is the zoom-in of Fig. $4 \mathrm{a}-\mathrm{c}$ in the momentum region around the positions of the Weyl nodes (the orange rectangle area shown in Fig. $4 a-c$ ). Figure $4 d$ clearly shows that the long topologically trivial arc in Fig. 4a lies far from the Weyl nodes in momentum space, and it is therefore not related to the Weyl nodes. With increasing energy, the topologically trivial arc moves toward the Weyl nodes and starts to touch the Weyl nodes at the Fermi level (Fig. 4e). In the constant energy contour of $E=+40 \mathrm{meV}$, the long trivial arc is disconnected with the Weyl nodes and a small topological Fermi arc (arc 2) can be seen in between the two Weyl nodes, as shown in Fig. $4 \mathrm{f}$ (also see Fig. S10). However, the separation in momentum space between the non-trivial arc (arc 2) and the trivial arcs (arc 1 and 3 ) is only about $0.008 \AA^{-1}$, which makes it extremely difficult to distinguish the scattering features in FT-STS induced by the non-trivial arc and those induced by the long trivial arc. Thus, by comparing the STM data with calculations, we conclude that the trivial and non-trivial scattering channels are difficult to distinguish in this compound.

Finally, our STM data show signatures of the broken inversion symmetry that's critical to the physics of $\mathrm{MoTe}_{2}$. Due to the bulk broken inversion symmetry, the two surfaces obtained after cleaving are inequivalent (Supplementary Fig. S11). As shown in Fig. S11, while the surface structure itself is the same, the atomic arrangement in the second layer is different between the two surfaces. An earlier ARPES study had suggested that this might affect the electronic structure. ${ }^{20}$ The two inequivalent surfaces were revealed in our data when different cleaves showed slightly different FT-STS patterns (Fig. 5, Supplementary Fig. S12 and Fig. S13). Our FT-STS calculations for the second surface, also show 
that the terminating electronic states are measurably different for the two surfaces. The differences arise from the different weights of bulk and surface states on the two terminations which makes the scattering channels in the measured FT-STS images taken on these two surfaces have different relative strengths. While the match between the calculations and data are not as good for this second surface, both STM as well as theory indicate that the broken inversion symmetry makes the two surfaces electronically inequivalent (Fig. 5).

In conclusion, we have performed a detailed low-temperature STM/STS study on the MoTe $\mathrm{T}_{2}$ sample. In the spatially resolved $\mathrm{d} / /$ $\mathrm{d} V$ maps, the quasiparticle interference patterns can be clearly resolved. We thoroughly characterized the electronic structure of $\mathrm{MoTe}_{2}$ with FT-STSs taken at energies both below and above the Fermi level. In the high-resolution FT-STSs above Fermi level, the scattering vectors between the trivial arcs and the projected bulk states can be clearly resolved. Due to the small momentum-space separation between the non-trivial arc and the trivial arc, it is difficult to clearly identify the non-trivial arc features in the FTSTSs. Our data show that the bulk broken inversion symmetry has a measurable effect on the surface electronic properties.

\section{METHODS}

\section{Sample synthesis}

The $\mathrm{MoTe}_{2}$ single crystals were grown by using a chemical vapor transport method. A mixture of stoichiometric Mo and Te powder were sealed into an evacuated quartz tube with iodine used as a transport agent. The quartz tube was then placed in a double zone furnace with temperature gradient from $900^{\circ} \mathrm{C}$ to $800^{\circ} \mathrm{C}$. Large-single crystals of centimeter size were obtained after 2 weeks. The composition and structure of $\mathrm{MoTe}_{2}$ single crystals were checked using X-ray diffraction and an energy-dispersive Xray spectrometer.

\section{Scanning tunneling spectroscopy measurements}

High-quality $\mathrm{MoTe}_{2}$ samples were cleaved at $77 \mathrm{~K}$ and immediately inserted into the STM scanner at $6 \mathrm{~K}$. Differential conductance (d//dV) spectra were acquired at $6 \mathrm{~K}$ using a standard lock-in technique with $\sim 4 \mathrm{mV}_{\text {rms }}$ modulation at a frequency of $987.5 \mathrm{~Hz}$.

Data availability

All data are available upon request from the corresponding authors.

\section{ACKNOWLEDGEMENTS}

STM work was supported by the US Department of Energy, Scanned Probe Division under Award Number DE-SC0014335. S.Y. acknowledges the financial support from Science and Technology Commission of Shanghai Municipality (STCSM) (Grant No. 18QA1403100) and the start-up funding from ShanghaiTech University. The work at Tulane University was supported by the US Department of Energy under Grant No. DE-SC0014208 (support for single crystal growth).

\section{AUTHOR CONTRIBUTIONS}

S.Y. and V.M. conceived the experiment. Samples were grown by J.H. and obtained from Z.M. S.Y. and D.I. carried out the STM studies. G.C., T.-R.C., and H.L. did all the calculations. S.Y., V.M., and D.I. wrote the paper.

\section{ADDITIONAL INFORMATION}

Supplementary information accompanies the paper on the npj Quantum Materials website (https://doi.org/10.1038/s41535-018-0112-5).

Competing interests: The authors declare no competing interests.

Publisher's note: Springer Nature remains neutral with regard to jurisdictional claims in published maps and institutional affiliations.

\section{REFERENCES}

1. Castro Neto, A. H. Charge density wave, superconductivity, and anomalous metallic behavior in 2D transition metal dichalcogenides. Phys. Rev. Lett. 86, 4382-4385 (2001).

2. Rossnagel, K. On the origin of charge-density waves in select layered transitionmetal dichalcogenides. J. Phys. Condens. Matter 23, 213001 (2011).

3. Sooryakumar, R. \& Klein, M. V. Raman scattering by superconducting-gap excitations and their coupling to charge-density waves. Phys. Rev. Lett. 45, 660-662 (1980).

4. Chang, T.-R. et al. Prediction of an arc-tunable weyl fermion metallic state in $\mathrm{Mo}_{\mathrm{x}} \mathrm{W}_{1-\mathrm{x}} \mathrm{Te}_{2}$. Nat. Commun. 7, 10639 (2016).

5. Soluyanov, A. A. et al. Type-II Weyl semimetals. Nature 527, 495-498 (2015).

6. Sun, Y., Wu, S.-C., Ali, M. N., Felser, C. \& Yan, B. Prediction of Weyl semimetal in orthorhombic MoTe 2 . Phys. Rev. B 92, 161107 (2015).

7. Wang, Z. et al. MoTe 2 : a type-Il Weyl topological metal. Phys. Rev. Lett. 117, 056805 (2016).

8. Huang, S.-M. et al. A Weyl fermion semimetal with surface fermi arcs in the transition metal monopnictide TaAs class. Nat. Commun. 6, 7373 (2015).

9. Weng, H., Fang, C., Fang, Z., Bernevig, B. A. \& Dai, X. Weyl semimetal phase in noncentrosymmetric transition-metal monophosphides. Phys. Rev. X 5, 011029 (2015).

10. Lv, B. Q. et al. Experimental discovery of Weyl semimetal TaAs. Phys. Rev. X 5, 031013 (2015).

11. Xu, S.-Y. et al. Discovery of a Weyl fermion semimetal and topological Fermi arcs. Science 349, 613-617 (2015).

12. Xu, S.-Y. et al. Discovery of Lorentz-violating type II Weyl fermions in LaAIGe. Sci. Adv. 3, e1603266 (2017).

13. Bruno, F. Y. et al. Observation of large topologically trivial Fermi arcs in the candidate type-II Weyl semimetal WTe 2 . Phys. Rev. B 94, 121112 (2016).

14. Beloposki, I. et al. Fermi arc electronic structure and Chern numbers in the type-II Weyl semimetal candidate $\mathrm{Mo}_{\mathrm{x}} \mathrm{W}_{1-\mathrm{x}} \mathrm{Te}_{2}$. Phys. Rev. B 94, 085127 (2016).

15. Deng, K. et al. Experimental observation of topological Fermi arcs in type-II Weyl semimetal MoTe 2 . Nat. Phys. 12, 1105-1110 (2016).

16. Feng, B. et al. Spin texture in type-II Weyl semimetal WTe 2 . Phys. Rev. B 94, 195134 (2016).

17. Huang, L. et al. Spectroscopic evidence for a type II Weyl semimetallic state in $\mathrm{MoTe}_{2}$. Nat. Mater. 15, 1155-1160 (2016).

18. Jiang, J. et al. Signature of type-II Weyl semimetal phase in MoTe ${ }_{2}$. Nat. Commun. 8, 13973 (2017)

19. Liang, A. et al. Electronic evidence for type II Weyl semimetal state in $\mathrm{MoTe}_{2}$. Preprint at https://arxiv.org/abs/1604.01706 (2016).

20. Tamai, A. et al. Fermi arcs and their topological character in the candidate type-II Weyl semimetal MoTe 2 . Phys. Rev. X 6, 031021 (2016).

21. Wang, $C$. et al. Observation of Fermi arc and its connection with bulk states in the candidate type-II Weyl semimetal WTe 2 . Phys. Rev. B 94, 241119 (2016).

22. Hoffman, J. E. et al. Imaging quasiparticle interference in $\mathrm{Bi}_{2} \mathrm{Sr}_{2} \mathrm{CaCu}_{2} \mathrm{O}_{8+\delta}$. Science 297, 1148-1151 (2002).

23. Berger, A. N. et al. Temperature-driven topological transition in $1 \mathrm{~T}^{\prime}-\mathrm{MoTe}_{2} . n p j$ Quantum Mater. 3, 2 (2018).

24. Deng, P. et al. Revealing fermi arcs and weyl nodes in $\mathrm{MoTe}_{2}$ by quasiparticle interference mapping. Phys. Rev. B 95, 245110 (2017).

25. Lin, C.-L. et al. Visualizing type-II Weyl points in tungsten ditelluride by quasiparticle interference. ACS Nano 11, 11459-11465 (2017).

26. Rüßmann, P. et al. Universal response of the type-Il Weyl semimetals phase diagram. Preprint at https://arxiv.org/abs/1706.00456 (2017).

27. Zheng, $\mathrm{H}$. et al. Atomic-scale visualization of quasiparticle interference on a typeII Weyl semimetal surface. Phys. Rev. Lett. 117, 266804 (2016).

28. Zhang, W. et al. Quasiparticle interference of surface states in the type-II Weyl semimetal $\mathrm{WTe}_{2}$. Phys. Rev. B 96, 165125 (2017).

Open Access This article is licensed under a Creative Commons Attribution 4.0 International License, which permits use, sharing, adaptation, distribution and reproduction in any medium or format, as long as you give appropriate credit to the original author(s) and the source, provide a link to the Creative Commons license, and indicate if changes were made. The images or other third party material in this article are included in the article's Creative Commons license, unless indicated otherwise in a credit line to the material. If material is not included in the article's Creative Commons license and your intended use is not permitted by statutory regulation or exceeds the permitted use, you will need to obtain permission directly from the copyright holder. To view a copy of this license, visit http://creativecommons. org/licenses/by/4.0/.

(c) The Author(s) 2018 